\title{
Les compléments prépositionnels dans les grammaires françaises de la première moitié du $\mathrm{XIX}^{\mathrm{e}}$ siècle
}

\author{
Piron, Sophie \\ Département de linguistique \\ Université du Québec à Montréal \\ piron.sophie@uqam.ca
}

\section{Introduction}

Cette recherche porte sur une trentaine de grammaires publiées au cours de la première moitié du $\mathrm{XIX}^{\mathrm{e}}$ siècle, essentiellement des grammaires scolaires, mais également quelques grammaires descriptives d'envergure. La fenêtre temporelle choisie commence avant 1823, c'est-à-dire avant la première grammaire scolaire (Chervel 1977), de manière à en saisir les prémices, et se clôt juste avant la seconde grammaire scolaire (en 1844, d'après Chervel 1977). Le corpus est constitué des grammaires suivantes : Fournier 1801 [1827], Boinvilliers (1802), Gaillard (1810 [1820]), Lhomond revu par Le Tellier (1811), Lemare (1807 [1819]), Girault-Duivier (1811 [1840]), Le Tellier (1816 [1822]), Guillon (1818), Noël et Chapsal (1823, 1827), Clouzet (1824), M. C. A (1825), Coffigny (1828), Delvart (1828), Boniface (1829 [1843]), Gaultier (1829), Thiel (1831), Farez (1832), Cornillac (1833), Bescherelle (1834 [1835 et 1852]), Bessière (1834), Cocquempot (1834), Clouzet (1835), Landais (1835 [1856]), Vanier (1836), Gilard (1837) et Perrot D'Ablancourt (1838).

L'objectif de cette recherche consiste à exposer avec précision comment la grammaire de la première moitié du XIX ${ }^{\mathrm{e}}$ siècle (telle qu'elle est représentée dans le corpus) traite les compléments prépositionnels, quel que soit le mot auquel ce complément s'attache. Les séquences suivantes seront analysées : nom syntagme prépositionnel (par exemple, la maison de mes parents), adjectif - syntagme prépositionnel (par exemple, content de soi), adverbe - syntagme prépositionnel (par exemple, beaucoup d'esprit) ${ }^{1}$ et verbe syntagme prépositionnel (par exemple, nuire à quelqu'un, aller à Lyon, combattre avec courage, être battu par quelqu'un).

Au-delà d'un relevé factuel, il s'agit d'analyser la conception que les grammairiens se sont faite de la préposition et des compléments qu'elle introduit. Cette conception est importante parce que la perspective adoptée par rapport à la préposition et aux fonctions dans lesquelles elle intervient constitue un marqueur de périodisation dans l'histoire de la grammaire française.

Comme l'a montré Chervel (1979), la deuxième grammaire scolaire naît en 1844 avec le complément circonstanciel. Celui-ci transformera la théorie grammaticale en lui faisant adopter une perspective rhétorique de l'énoncé, mais aussi en y réduisant le rôle de la préposition (Chevel parle à ce sujet de "déchéance de la préposition », 1979, p. 19). Il nous apparaissait dès lors intéressant d'étudier avec précision comment la première grammaire scolaire traite la préposition et les compléments prépositionnels.

Cet article envisage d'abord comment les grammairiens de la première moitié du XIX ${ }^{\mathrm{e}}$ siècle ont défini la préposition et la notion de complément, il envisage ensuite comment les syntagmes prépositionnels ont été analysés dans différentes configurations, à savoir en complément d'un nom, d'un adjectif, d'un adverbe et d'un verbe. Il détache enfin quelques constructions au sein des séquences verbe - syntagme prépositionnel. Il s'agit de constructions dont l'analyse subira, au fil des décennies suivantes, des changements profonds. 


\section{La préposition}

La définition de la préposition au sein de ces grammaires a une incidence sur l'analyse des syntagmes prépositionnels. Les définitions trouvées dans le corpus de grammaires présentent deux perspectives : la première consiste à analyser la préposition comme un maillon entre deux unités, la seconde à la voir comme un élément introduisant une unité. Dans les deux cas, l'approche peut être tantôt grammaticale tantôt logique.

Presque la moitié des grammairiens du corpus proposent une définition de la préposition en combinant les deux perspectives, c'est-à-dire celle d'une relation ternaire, où la préposition est définie comme lien entre deux mots, et celle d'une relation binaire, où la préposition est définie comme terme recteur. On trouve ainsi les auteurs suivants : Reymond 1811, Guillon 1818, Le Tellier 1816, Gaillard 1820, Noël et Chapsal NGF 1823 [1831], Coffigny 1828, Farez 1832, Cornillac 1833, Bessière 1834, Cocquempot 1834, Landais 1835 [1856].

(1) « La préposition établit et exprime un rapport entre deux mots [...] Le second mot ou terme de ce rapport, mot qui suit toujours immédiatement la préposition, a été appelé complément de la préposition.»(Bessière, 1834, p. 205)

\subsection{La préposition comme lien}

Parmi les grammairiens qui définissent la préposition comme une partie du discours permettant d'établir un lien entre deux mots (soit une séquence $<\mathrm{X}$ Préposition $\mathrm{X}>$ ), certains envisagent ce lien sur le plan de l'analyse grammaticale. La préposition est alors la marque - presque morphologique - d'une relation syntaxique, voire d'un cas. Dans cette perspective, la plupart des auteurs ci-dessous font usage du verbe marquer lorsqu'ils définissent le rôle de la préposition.

(2) «La préposition est un mot invariable qui se place devant les noms ou pronoms, et qui marque les différents rapports que les choses ont entre elles. » (Fournier, 1801 [1827], p. 83)

(3) «Elle marque les rapports ou les différentes relations que les choses ont entr'elles [...]. » (Reymond, 1811, p. 144)

(4) « la préposition sert à marquer les différents rapports que les choses ont entr'elles » (Guillon, 1818, p. 118)

(5) « sert à marquer le rapport des choses entr'elles. » (Gaillard, 1820, p. 66)

(6) «Sert à marquer les rapports que les choses ont entr'elles.» (Le Tellier, 1816 [1822], p. 100)

(7) «La fonction de la préposition est de marquer le rapport qu'il y a entre deux mots [...]. » (Noël et Chapsal, LAG, 1827 [1871], p. 13, par. 40)

(8) « La préposition sert à marquer un rapport entre deux termes » (M.C.A., 1825, p. 47)

(9) «La préposition est un mot qui marque les rapports que les personnes ou les choses ont entre elles » (Coffigny, 1828, p. 96)

(10) La préposition « indique seulement le cas » (Gaultier, 1829, p. 74)

(11) «Les prépositions servent à marquer le rapport d'un nom à un autre mot» (Thiel, 1831, [1836], p. 13)

(12) «Les prépositions servent à marquer le rapport que les mots ont entre eux. » (Farez, 1832, p. 51)

(13) «La préposition est un mot invariable qui sert à marquer les rapports que les mots ont entre eux. »(Cornillac, 1833, tableau de la préposition)

(14) «La préposition est un mot qui sert à joindre le nom ou pronom suivant au mot qui la précède. » (Cocquempot 1834, p. 96) 
Lorsque la préposition est conceptualisée au sein d'une séquence $\langle\mathrm{X}$ Préposition $\mathrm{X}\rangle$, d'autres grammairiens envisagent le lien établi non plus sur le plan grammatical, mais sur celui de l'analyse logique, c'est-à-dire que la relation instaurée par la préposition relève, dans cette conception, de la signification. Il s'agit de Boinvilliers (1802), Noël et Chapsal (1823), Boniface (1829), Bescherelle (1834), Vanier (1836) et D'Ablancourt (1838). Ainsi, certains grammairiens utilisent le mot exprimer dans leur définition de la préposition. Le choix de ce verbe semble insister sur le fait que la préposition est un indicateur du lien sémantique existant entre les mots qu'elle relie. Il n'est alors pas inhabituel de trouver dans le chapitre dédié aux prépositions un classement selon leurs différentes significations de lieu, de temps, de cause, etc. Ces notions sont précisées par le complément que la préposition introduit.

$\mathrm{Au}$ sein de l'analyse centrée sur la signification, on relèvera plusieurs prises de position sur la valeur sémantique de la préposition. La majorité des auteurs semblent prendre parti pour un sens plein ou, à tout le moins, fort.

(15) «La préposition est un mot invariable qui sert à exprimer les rapports que les mots ont entre eux. » (Noël et Chapsal, NGF, 1823 [1831], p. 68)

(16) «puisque l'esprit saisit des rapports, soit entre les objets, soit entre les qualités ou les actions de ces mêmes objets, il faut nécessairement dans les langues une espèce de mots qui soient signes de ces rapports, qui les indiquent. » (Bescherelle, 1834 [1852], p. 773)

(17) «Partie indéclinable du discours qui exprime un rapport d'idée à idée, dont la première s'appelle l'antécédent et la seconde le conséquent. [...] Dans Paul va à Paris, la préposition à met en rapport le verbe va avec Paris. » (Vanier 1836, p. 555) «Comme les idées de rapports entre l'antécédent et le conséquent, ne sont pas toujours facilement saisies par les jeunes intelligences, on peut dire aux adolescents que l'accent grave placé sur le mot à, préposition, est mis là comme une petite flèche qui indique l'objet vers lequel se porte l'action [...].Tout le monde sentira que dans il va, on exprime une action; mais qu'en ajoutant à Paris, ou à Bordeaux, ou enfin à quelque endroit que ce soit, le nom de la ville ou de l'endroit est évidemment le point vers lequel l'action se dirige [...]. » (Vanier 1836, p. 2)

(18) «La préposition est un mot invariable qui sert à exprimer les rapports que les mots peuvent avoir entre eux. » (D'Ablancourt, 1838, p. 53)

D'autres auteurs soulignent la signification incomplète de la préposition, qui a alors besoin de son complément.

(19) «Le déterminatif (ce mot remplace celui de préposition, qui est vide de sens) est un mot invariable, qui lie deux termes, en attachant au premier une idée incomplète, qu'achève le second. » (Boinvilliers, 1802, p. 59)

(20) «Dans Il parle avec facilité, il est affable envers tout le monde, les mots avec et envers modifient, l'un le verbe parle, et l'autre l'adjectif affable: il parle avec, il est affable envers; mais comme, pour l'expression totale de la pensée, ces mots ont besoin d'un complément, on peut dire qu'ils modifient d'une manière incomplète. [...] La préposition est donc un mot qui modifie le verbe et l'adjectif d'une manière incomplète. » (Boniface, 1829 [1843], p. 45-46)

Enfin, Clouzet va jusqu'à nier toute signification à la préposition.

(21) «La Préposition est un mot qui ne signifie rien par lui-même; mais qui, avec son complément (c'est-à-dire avec le substantif, le pronom, ou l'infinitif qui le suit) exprime le rapport qui existe entre ce complément et ce qui précède. » (Clouzet, 1824, $2^{\mathrm{e}}$ tableau, colonne 7)

Il est intéressant de constater que Noël et Chapsal utilisent le verbe marquer pour définir la préposition dans les Leçons d'analyse grammaticale, où la perspective adoptée est, forcément, grammaticale ( $\mathrm{La}$ fonction de la préposition est de marquer le rapport qu'il y a entre deux mots » Noël et Chapsal, 1827 [1871], p. 13, par. 40). À l'inverse, ces mêmes auteurs définissent la préposition sous un angle sémantique dans la Nouvelle grammaire française, et emploient le verbe exprimer («La préposition est un mot 
invariable qui sert à exprimer les rapports que les mots ont entre eux. » Noël et Chapsal, 1823 [1831], p. 68).

Les deux approches - logique et grammaticale - peuvent être unifiées dans la définition, et présenter à la fois le lien syntaxique (établir, désigner) et le lien sémantique (exprimer). La signification de la préposition y est considérée comme complète.

(22) «La préposition établit et exprime un rapport entre deux mots [...].» (Bessière 1834, p. 205)

(23) «Les prépositions sont des mots qui servent à exprimer ou à désigner les différents rapports que les choses ont les unes aux autres. » (Landais, 1835 [1856], p. 364a-b)

\subsection{La préposition comme introducteur}

Lorsque la préposition est définie comme une partie du discours appelant un autre mot (soit la séquence $<$ Préposition $\mathrm{X}>$ ), les grammairiens repèrent et isolent une suite de mots, qu'ils définissent tantôt syntaxiquement, tantôt sémantiquement. Ainsi, les grammairiens suivants définissent-ils l'unité formée par la préposition et le terme qu'elle introduit sous l'angle grammatical de la rection ou du complément.

(24) «La préposition elle-même se distingue par son régime »(Guillon, 1818, p. 118)

(25) «Les prépositions ont un complément. » (Le Tellier 1816[1822], p. 100)

(26) «Les prépositions sont toujours suivies d'un complément ou régime. » (Farez, 1832, p. 51)

(27) «Le fruit de l'arbre, utile à l'homme. [...] De, à sont des prépositions; le mot qui suit s'appelle le régime de la préposition. »(Cocquempot 1834, p. 97)

(28) «Elles [les prépositions] sont suivies d'un régime. » (Landais, 1835 [1856], p. 365a)

Mais la séquence $<$ Préposition $X>$ peut également être conceptualisée sur le plan de l'analyse logique, c'est-à-dire que le mot appelé complète le sens de la préposition.

(29) «D. Comment appelle-t-on les mots que les prépositions précèdent ? R. Complément, parce qu'il complète leur sens. » (Gaillard, 1820, $3^{\mathrm{e}}$ éd., p. 66)

(30) «Les prépositions n'ont par elles-mêmes qu'un sens incomplet; le mot qui en complète la signification est le régime de la préposition » (Noël et Chapsal, NGF, 1823 [1831], p. 68)

(31) «La préposition n'a par elle-même qu'un sens incomplet; il faut qu'elle soit suivie d'un autre mot qui en complète la signification : ce mot est appelé régime de la préposition. »(Cornillac, 1833, tableau de la préposition)

Les perspectives grammaticale (présence d'un régime) et logique (la préposition est dépourvue de sens si elle est seule) peuvent coïncider dans un même ouvrage.

(32) «ce qui la [la préposition] fait principalement connaître, c'est qu'elle a toujours un régime ou complément, sans lequel elle n'aurait point de sens. » (Reymond, 1811, p. 146)

(33) «La préposition [...] a besoin d'un régime pour avoir un sens complet. » (Coffigny, 1828, p. 96)

\subsection{Les tendances}

Il ressort de ce panorama sur la préposition que les grammairiens de la première moitié du XIX ${ }^{\mathrm{e}}$ siècle dans notre corpus optent toujours pour une définition ternaire de la préposition. Certains s'y limitent (Fournier 1801, Boinvilliers 1802, Clouzet 1824, M.C.A. 1825, Boniface 1829, Gaultier 1829, 
Bescherelle 1834, Thiel 1836, Vanier 1836, D'Ablancourt 1838), tandis que d'autres recadrent ensuite la définition sur une relation binaire entre la préposition et son régime.

Quelle que soit la perspective adoptée, la préposition est plus souvent envisagée sur le plan grammatical, comme lien ou comme introducteur. Lorsque la préposition est vue à travers une analyse de la signification, son sens n'est considéré comme complet que lorsque les grammairiens adoptent une perspective ternaire. Par contre, la nécessité d'avoir un complément pour saturer le sens de la préposition se trouve autant dans une analyse ternaire que binaire.

\section{La notion de régime et de complément}

Les grammaires du corpus ne définissent pas systématiquement la notion de régime ou de complément en tant que concept structurant. De plus, les quelques ouvrages où l'on trouve une telle définition ne transposent pas à l'étude de la complémentation les réflexions liées à la préposition. On trouve ainsi des définitions très générales :

(34) «Le régime ou complément est le mot qui complète le verbe actif ou la préposition. » (Clouzet, 1835, p. 63)

Sinon, les grammairiens adoptent une approche fondamentalement sémantique et linéaire (« une relation de mot à mot » Chervel 1979, p. 18), celle de la complétude du sens.

(35) «D. Qu'appelle-t-on complément ou régime dans la phrase ? R. Le complément sert à compléter, à étendre, à développer le sens d'une préposition, ou à le fixer sur un sujet particulier. » (Reymond, 1811, p. 178)

(36) «On appelle, en général, Régime ou Complément un mot qui achève d'exprimer, qui complète l'idée commencée par un autre mot. »(GiraultDuvivier, 1811 [1840], T1, p. 675)

(37) Les modifications « sont les mots qui modifient ou qualifient le sens des noms ou des verbes de la phrase. » (Gaultier, 1829, p. 118)

(38) «Tout ce qu'on ajoute à un mot principal pour compléter l'expression de l'idée qu'on veut rendre, s'appelle complément. » (Thiel, 1831 [1836], p. 59)

(39) «On appelle régime le mot qui dépend immédiatement d'un autre mot et qui sert à en compléter la signification. » (Cornillac, 1833, tableau du verbe)

(40) «Le complément [...] n'est autre chose que des mots qu'on ajoute à un mot pour en compléter ou en déterminer la signification, de quelque manière que ce puisse être. » (Landais, 1835 [1856], p. 392b)

Les auteurs ont plutôt tendance à définir cette notion au sein des chapitres consacrés aux parties du discours acceptant un complément.

\section{La construction nom - syntagme prépositionnel}

Les constructions du type nom - syntagme prépositionnel comme la maison de mes parents ne sont pas systématiquement analysées dans les grammaires de l'époque, qui s'en préoccupent peu d'un point de vue fonctionnel et ne les abordent souvent que sous un angle normatif en insistant sur le choix du nombre pour le nom introduit par la préposition.

Certains grammairiens adoptent une analyse binaire, qui n'envisage que la préposition et le mot suivant, soit une séquence $<$ Préposition $\mathrm{x}>$. Ils considèrent alors que le substantif est le régime de la préposition. Ainsi, pour Lemare (1804, éd. 1835) dans «La grandeur de Rome parut bientôt dans ses édifices publics » (T1, par. 1003), Rome est le régime de la préposition de; pour Cornillac (1833), dans le fruit de l'arbre, arbre est régime de la préposition de (tableau de la préposition); pour Bessière (1834), dans la bonté de Dieu, Dieu est le « complément de la préposition » (p. 205). Bescherelle (1834, éd. 1852) aborde dans la section «Du nombre des substantifs, compléments d'une préposition » des constructions telles que «peaux de léopard; branches de laurier; pommiers en fleurs » (p. 128). 
D'autres grammairiens adoptent une anlyse ternaire, soit la séquence $<\mathrm{x}$ Préposition $\mathrm{x}\rangle$, puisqu'ils considèrent que le nom introduit par la préposition est le régime ou complément du substantif précédent. Ainsi, pour Le Tellier revisitant Lhomond (1811), «Un substantif ne peut être régime d'un autre substantif, qu'à l'aide d'une préposition : la beauté de l'univers; moulin à vent. Drogue pour drogue. »" (p. 98). Girault-Duvivier (1811) considère la phrase «A peine nous sortions des portes de Trézène » (T2, p. 1155) et indique « Trézène, [...] régime du substantif portes » (T2 p. 1156).

L'analyse des séquences nom - syntagme prépositionnel ne se présente donc pas systématiquement comme ternaire, contrairement à la définition de la préposition. De plus, certains ouvrages semblent préciser que le syntagme prépositionnel forme un ensemble ayant une fonction. Ils dépassent ainsi l'analyse linéaire et présentent une analyse binaire, certes, mais sous la forme d'un regroupement des termes $<\mathrm{X}$ [Préposition $\mathrm{X}]>$ et d'une hiérarchisation des constituants :

(41) «Les richesses de la nature sont innombrables. Cette proposition est modifiée ou complexe dans son sujet, les richesses de la nature; sont en est le verbe, et innombrables, l'attribut. Richesses en est le sujet grammatical; de la nature en est le complément déterminatif. » (Boniface 1829, éd. 1843, p. 111)

(42) «la religion de ce peuple est inconnue, de ce peuple est le complément déterminatif de religion. » (Vanier 1836, p. 132).

Landais propose une analyse hiérarchisée : dans La manufacture de Maubeuge, «Maubeuge est le complément particulier de la préposition de; de Maubeuge [...] est le complément du substantif manufacture. » (Landais 1835 [1856], p. 393b).

Enfin, on trouve dans certaines grammaires de cette époque une appellation identique pour désigner le syntagme prépositionnel accroché à un autre mot, quelle qu'en soit la nature (nom, adjectif ou verbe) : il s'agit du régime ou complément indirect.

(43) [Complément prochain indirect] Les charmes de l'étude (Boinvilliers, 1802, T1, p. 16)

(44) «Le substantif et le pronom ne peuvent avoir qu'un complément indirect.» (Noël Et Chapsal, 1827 [1871], LAG, p. 5, par. 15)

(45) «dans table de marbre, de marbre est le compl. indirect de table. » (Noël et Chapsal, 1827 [1871], LAG, p. 13, par. 40)

(46) «les compléments des noms peuvent aussi être regardés comme des compléments indirects; ils sont ordinairement marqués par $d e$. La justice de Dieu. L'intérêt de l'homme. Le temps d'exécuter son projet audacieux. » (Thiel, 1831 [1836], p. 64)

Les grammaires du corpus présentent donc des variations dans leur vison de la complémentation d'un substantif. Dans tous les cas, cependant, l'analyse relève du plan grammatical et non de celui de la signification (c'est-à-dire de la complétude du sens).

\section{La construction adjectif - syntagme prépositionnel}

La construction du type adjectif - syntagme prépositionnel (par exemple, bon envers ses parents) suscite davantage d'intérêt chez les grammairiens de l'époque, en particulier d'une part, pour la liste des adjectifs autorisant cette construction et, d'autre part, pour la restriction sur le choix de la préposition. Les grammairiens citent le plus fréquemment les prépositions à et de, mais certains ouvrent la liste à beaucoup plus d'emplois (ainsi, M.C.A. 1825). Les auteurs usent des termes régime et complément. Le second ne bénéficie pas encore du ralliement de tous les grammairiens.

L'analyse de la complémentation de l'adjectif présente trois angles d'approche. Le premier consiste en la vision de la dépendance immédiate, qui considère qu'un mot est régi par une préposition. L'analyse est binaire et se présente sous la forme d'une séquence $<$ Préposition $\mathrm{X}>$. 
(47) «je suis disposé à étudier [...] complément ou régime de la préposition » (Clouzet, 1835, p. 63)

Le deuxième angle d'analyse considère une chaîne de trois unités $(<$ Adjectif Préposition $\mathrm{X}>$ ), posant alors qu'un mot est régime d'un autre et que la préposition joue le rôle de lien.

(48) «Le régime des adjectifs est un substantif ou un verbe. [...] On met de ou à entre cet adjectif et le substantif, ou le verbe. Exemples. Digne de récompense, propre à la guerre; un enfant chéri de son père; un homme habile à tirer de l'arc, etc. » (Lhomond, 1807, p. 119)

(49) «Ce complément verbe, ou nom, s'appelle régime des adjectifs. Le rapport du complément avec l'adjectif s'exprime par une de ces prépositions $\grave{a}$, de, dans, en, sur, etc. » (M.C.A., 1825, p. 15)

(50) «digne de récompense; content de son sort; utile à l'homme. Récompense est le régime de l'adjectif digne, parce qu'il est joint à cet adjectif par le mot de. » (Coffigny, 1828, p. 24)

(51) «Digne de récompense, content de son sort, utile à l'homme, semblable à son père, propre à la guerre. [...] Récompense est le régime de l'adjectif digne, parce qu'il est joint à cet adjectif par le mot de. »(Cocquempot, 1834, p. 24)

(52) «Complément d'un adjectif» : « le complément est précédé de la préposition à » (Bescherelle, 1834 [1835], p. 234) «contraire à lui-même, sujet au repentir» (Bescherelle, 1834, [1835], p. 235)

La troisième approche est binaire, mais envisage l'ensemble formé par la préposition et le mot qu'elle introduit comme un complément, soit la séquence $<$ Adjectif [Préposition X] $>$.

(53) «Le régime ou complément des adjectifs est un substantif ou un verbe précédé de l'une des prépositions $\grave{a}$, de, dans, en, sur, etc. » (Girault-Duvivier, 1811 [1840], T1, p. 293)

(54) «Le régime ou complément des adjectifs est un substantif ou un verbe avec une préposition.» (Landais, 1835 [1856], p. 459a); «adapté aux circonstances; utile à sa patrie, digne de respect, facile à concevoir, etc., etc., aux circonstances, à sa patrie, de respect, à concevoir, sont autant de compléments des adjectifs : adapté, utile, digne, facile, etc. » (Landais, 1835 [1856], p. 393a)

(55) «Dans : il est bon envers les pauvres, ces mots envers les pauvres forment un complément attributif. » (Vanier, 1836, p. 132)

Signalons enfin que les grammairiens qui analysent uniformément tous les compléments de type prépositionnel les qualifient de régimes indirects ou de compléments indirects en général, et bien sûr en particulier lorsqu'ils se rapportent à un adjectif.

(56) «Régime indirect [...] Je suis sensible aux preuves d'amitié que vous m'avez données, et j'en suis content. » (Noël et Chapsal, NGF, 1823 [1831], p. 138139)

(57) «L'adjectif ne peut avoir également qu'un complément indirect. » (Noël et Chapsal, LAG, 1827 [1871], p. 6, par. 17)

(58) «Les compléments des adjectifs qualificatifs, toujours liés à ces adjectifs par $\grave{a}$, de, en, dans, sur, etc., sont aussi des compléments indirects. Content de son sort. Modeste en ses désirs. Utile à l'homme. Funeste aux nations. " (Thiel, 1831 [1836], p. 64)

(59) «Quelques grammairiens n'appliquent pas seulement le mot complément aux verbes et aux prépositions, comme nous venons de le voir, ils l'appliquent encore aux substantifs et aux adjectifs. (Vanier, 1836, p. 132)

Dans tous les cas, l'analyse de la séquence adjectif - syntagme prépositionnel est envisagée d'un point de vue grammatical, et non sur le plan de la signification, c'est-à-dire de la complétude du sens. 


\section{La construction adverbe - syntagme prépositionnel}

Cette construction est délaissée par les grammaires, excepté par Noël et Chapsal (1823 [1831]), qui en proposent une analyse réduite, mais alignée sur le reste du système. Dans cette optique, toute préposition liant un terme à un adverbe (de quantité) donne lieu à un complément indirect. Ici encore, l'insistance se pose sur la construction et non sur la complétude du sens.

(60) L'adverbe de quantité, comme beaucoup, peu, guère, moins, plus, autant, trop, etc., faisant l'office de substantif, est susceptible, comme celui-ci, d'avoir un complément indirect exprimé par un substantif ou un pronom précédé de la préposition de: beaucoup d'esprit, bien des amis, peu du sien, moins de celui$c i$, exemples dans lesquels d'esprit est le complément de l'adverbe beaucoup [...]. » (Noël et Chapsal, LAG, 1827 [1871], p. 6, par. 16)

\section{La construction verbe - syntagme prépositionnel}

La construction du type verbe - syntagme prépositionnel a fait l'objet d'une attention soutenue de la part des grammairiens. Rares sont les grammairiens qui envisagent cette construction comme une relation binaire dont le verbe est exclu. Dans ce cas, seule compte la relation entre la préposition et le terme qu'elle régit.

(61) «On ne pourrait donc pas dire que Rome, dans donne ton sang à Rome, et dans gens qui viennent de Rome, Rome est le régime indirect de donne et de viennent. [...] Dans donne ton sang à Rome, Rome est régi par à, il est sous sa dépendance immédiate; c'est donc de cet invariable qu'il est complément essentiel; il ne l'est ni directement ni indirectement du verbe donne. » (Lemare 1804 [1835], T1, par. 1005)

Clouzet (1824), après avoir proposé la suite de questions permettant de repérer le régime indirect, précise que «dans l'analyse grammaticale, il vaut mieux les désigner comme Régimes de la préposition qui les précède. » (Clouzet 1824, $3^{\mathrm{e}}$ tableau, colonne 2). Certains grammairiens réduisent même cette relation à la seule présence de la préposition et indiquent que le régime est simplement marqué au moyen des prépositions (Lhomond, 1811).

De manière générale, il n'apparaît pas toujours clairement comment les grammairiens envisagent la construction verbe - syntagme prépositionnel. Il semble que la majorité d'entre eux la conçoivent comme une relation binaire entre le verbe et un complément (constitué d'une préposition et d'un substantif), mais les explications de certains d'entre eux pointent vers une analyse ternaire où un verbe est lié à un autre élément au moyen d'une préposition. Contrairement aux séquences des sections précédentes (nom, adjectif ou adverbe suivis d'un syntagme prépositionnel), l'angle d'approche est désormais plutôt sémantique : le verbe est sémantiquement complété par un élément.

(62) «Le complément [...] indirect est le mot vers lequel l'expression se dirige [...], mais avec le secours d'un mot intermédiaire, qui lui fait prendre une voie oblique [...]. » (Boinvilliers, 1802, T1, p. 16)

La complétude de la signification intervient dans la définition du régime ou complément indirect, mais elle n'apparaît qu'au détour des questions qu'il faut poser mécaniquement. Celles-ci reposent sur l'identification d'une personne (qui?) ou d'une chose (quoi?). La majorité des grammairiens du corpus les utilisent, et ce, uniquement pour les verbes (Reymond 1811, Girault-Duvivier 1811[1840], Guillon 1818, Gaillard 1820, Le Tellier 1816[1822], Noël et Chapsal 1823 [1831], Clouzet 1824, M.C.A 1825, Fournier 1801 [1827], Boniface 1829[1843], Cornillac 1933, Gilard 1837, D'Ablancourt 1838). Il s'agit de la tradition instaurée par Restaut (1732).

La liste des prépositions citées varie d'un ouvrage à l'autre et ouvre les possibilités de significations. Une des particularités de l'époque est de proposer des listes relativement longues, et non exhaustives. Il faut en déduire que la complémentation n'est pas restreinte à quelques prépositions (par exemple, à et $d e$ ) ni à quelques significations (par exemple, l'objet d'une action). Ce qui prévaut au cours de la première moitié 
du XIX ${ }^{\mathrm{e}}$ siècle est la complétude du sens, quel qu'il soit. Le principe mis de l'avant consiste à utiliser les mots interrogatifs qui et quoi, et de les faire précéder d'une préposition. L'intérêt d'un tel procédé est d'être bâti sur le lien que la préposition établit entre deux termes, et donc sur une relation ternaire. Des questions telles que dans quoi? sur quoi? (et non où ?) permettent ainsi de préserver la tripartition $<$ Verbe Préposition $X>$, ou au moins la conception linéaire, même si les auteurs semblent analyser en un seul constituant la préposition et le mot qu'elle introduit.

(63) «Le régime composé ne vient jamais en réponse directe à la question : qui ? ou quoi ? Si l'on veut le découvrir par le moyen d'une question, il faut que cette question renferme une préposition, comme : à qui ? à quoi ? de qui ? par quoi ? sur quoi ? dans quoi ? etc. » (Reymond 1811, p. 98)

(64) «Le Régime indirect est celui qui complète indirectement l'idée commencée par le verbe, c'est-à-dire qu'il ne la complète qu'à l'aide d'une préposition exprimée ou sous-entendue; il est le terme de l'action que le verbe exprime, et répond aux questions à qui ? de qui ? pour qui? par qui ? etc., pour les personnes; à quoi ? pour quoi ? de quoi ?, etc., pour les choses. » (GiraultDuvivier, 1811, T1, p. 676)

(65) «Le régime indirect est celui qui complète la signification du verbe à l'aide de certains mots qu'on appelle préposition; tels sont $\grave{a}$, de, pour, avec, dans, etc. Il répond à l'une des questions à qui? de qui ? pour qui ? avec qui ? etc., pour les personnes, et à l'une de celles-ci, à quoi ? de quoi ? pour quoi ? avec quoi ? pour les choses. » (Noël et Chapsal, NGF, 1823 [1831], p. 22)

(66) «Le régime indirect d'un Verbe est celui qui complète l'affirmation du Verbe à l'aide d'une préposition; le régime indirect répond à la question $\grave{a}$ qui ? à quoi ? par qui ? par quoi ? de qui ? de quoi ? placée après le Verbe [...].» (Clouzet, 1824, 2e tableau, colonne 5)

(67) Le régime indirect est celui qui complète la signification du verbe au moyen d'un mot intermédiaire, tels que $\grave{a}$, pour, de, avec, dans, etc.; il répond à l'une des questions à qui ? de qui ? pour qui ? avec qui ? pour les personnes; et à quoi ? de quoi ? pour quoi ?, etc. pour les choses » (Bescherelle, 1834 [1852], p. 492)

(68) « On appelle régime indirect le nom ou pronom qui donne à l'action du verbe une idée plus complète, ce qui a lieu à l'aide des prépositions $a$, $a u$, de, avec, dans, sans, vers, etc. Il répond aux questions à qui ? de qui ? pour qui ? avec qui ? sans qui ? vers qui ? en parlant des personnes; et de quoi ? à quoi ? pour quoi ? avec quoi ? en parlant des choses. » (D'Ablancourt 1838, p. 21)

D'autres grammaires proposent la question où et même la question comment. Ils gomment alors l'uniformité de l'analyse, celle-ci n'étant plus composée de la séquence de trois termes <Verbe Préposition qui / quoi $>$.

(69) « [Le régime indirect,] C'est le nom qui est précédé d'une préposition, et qui répond à l'une de ces questions, à qui, à quoi; de qui, de quoi; d'où; par qui, par quoi, comment, où, par où. » (Gaillard 1820, p. 93)

(70) «Le régime indirect est celui qui complète la signification du verbe, à l'aide de certains mots, qu'on appelle prépositions; tels sont : à, de, avec, par, etc. On connaît le régime indirect, en mettant après le verbe une des questions à qui, à quoi, de qui, de quoi, où, d'où, par qui ou par quoi. » (Cornillac 1833, tableau du verbe)

(71) «Le complément indirect répond aux questions à qui ? à quoi? comment? quand ? où? par quel motif? en un mot, qui ou quoi précédés d'une préposition quelconque. »(Gilard 1837, p. 83)

Si le mécanisme des questions n'est pas présenté, la longue liste des prépositions, où l'on trouve des prépositions de lieu, ne laisse aucun doute sur le sens locatif (concret ou abstrait) qu'elles introduisent, et donc sur la grande variété de sens que peut prendre le complément indirect. 
(72) « complément appelé indirect [...] est uni [au verbe] par une des prépositions à, de, par, pour, vers, sur, sous, dans, en, hors, etc. » (Thiel 1831, p. 63)

(73) «Ce que nous appelons communément régime indirect, et que d'autres appèlent plus logiquement le terminatif, que nous rendons par les prépositions $\grave{a}$, dans, vers, sur, etc. [...]. » (Vanier 1836, p. 130)

Cependant, la liste des prépositions est parfois restreinte aux prépositions $\grave{a}$, de et par.

(74) «Le régime composé ou indirect se place aussi après le verbe; mais il est précédé des mots à de ou par, et répond aux questions à qui, de qui, par qui, à quoi, de quoi, par quoi ?» (Fournier 1801 [1827], p. 67) « Le complément est indirect, quand il est séparé du verbe par une des trois prépositions $\grave{a}$, de, par. » (Fournier 1801 [1827], p. 89-90)

(75) «Tout complément d'un verbe est indirect, s'il répond à l'une des questions à qui ? à quoi ? de qui ? de quoi ? par qui ? par quoi ?» (Boniface 1829 [1843], p. 30)

Certaines grammaires réduisent la liste aux deux prépositions à et $d e$.

(76) «Le complément indirect est l'objet sur lequel l'action marquée par le verbe ne tombe qu'indirectement. Il est toujours précédé des prépositions $a ̀$ et $d e$.» (Le Tellier 1816[1822], p. 74)

(77) «Le verbe actif peut toujours avoir un régime direct. Il peut aussi avoir un régime indirect, marqué par une préposition $\grave{a}$, de. » (Farez 1832, p. 28)

(78) «Ce régime est précédé de la préposition à, s'il s'agit du but où tend l'action; et de la préposition de, s'il s'agit du terme d'où cette action vient ou commence. » (Landais 1835 [1856], p. 518a)

Or, dans les compléments ou régimes indirects donnés en exemples, le lecteur pourra trouver des syntagmes prépositionnels de lieu, qui ne peuvent correspondre en toute vraisemblance à une question en à quoi ? ou de quoi?

(79) «Le régime indirect ou relatif, est le nom ou le pronom qui est séparé du verbe par de ou $\grave{a}$. [...] On connaît le régime indirect à l'une des questions de qui ? de quoi ? à qui ? à quoi ? [...] Il exprime une chose qui n'a qu'un rapport indirect avec l'action ou la signification du verbe. » Dans aller à la campagne, « campagne est le régime indirect ou relatif du verbe aller. » (Guillon 1818, p. 98)

Dans tous les cas, un élément introduit par une préposition et indiquant le lieu est analysé comme un régime ou un complément indirect (Boinvilliers 1802, Lemare 1804, Girault-Duvivier 1811, Guillon 1818, Gaillard 1820, Coffigny 1828, Delvart 1828, Gaultier 1829, Thiel 1831, Clouzet 1835, Gilard 1837). En effet, le sens n'est envisagé que sous l'angle de la complétude. À cette époque, aucune signification (le lieu, par exemple) n'entraîne une analyse différente. La construction syntaxique reste le principe déterminant pour l'analyse du complément.

Boinvilliers (1802) fait figure d'exception en différenciant la relation sémantique nécessaire de celle qui est facultative :

(80) «Le complément prochain indirect est le mot vers lequel l'expression se dirige nécessairement, mais avec le secours d'un mot intermédiaire, [...].» (Boinvilliers, 1802, T1, p. 16) «Le complément éloigné indirect est le mot vers lequel l'expression se dirige accidentellement, et avec le secours d'un mot intermédiaire [...]. » (Boinvilliers, 1802, T1, p. 19)

Une autre voie d'analyse, sémantique elle aussi, côtoie la liste des questions. Les grammairiens l'empruntent en parallèle. Ils proposent alors d'évaluer, semble-t-il, le rapport sémantique entre le verbe et le syntagme prépositionnel. L'analyse quitte le niveau linéaire des mots. C'est uniquement sous cet angle que l'on voit apparaître une opposition entre complément indirect et complément circonstanciel. Dans le corpus étudié ici, les ouvrages faisant référence au circonstanciel pour traiter de syntagmes 
prépositionnels sont ceux de Girault-Duvivier 1811 [1840], Noël et Chapsal (1823 et 1827), Thiel (1831) et Vanier (1836). Quant à Boniface (1829 [1843], il utilise le terme de complément adverbial.

Certaines séquences $<$ Préposition $\mathrm{X}>$ sont considérées comme un terme de l'action (Girault-Duvivier 1811, Landais 1835, Vanier 1836) ou comme l'origine de celle-ci (Girault-Duvivier 1811, Landais 1835). Elles reçoivent l'appellation de complément indirect.

(81) « Ce qui doit marquer le but auquel aboutit l'affirmation ou celui duquel elle part, présente naturellement un Terme. Il est le complément indirect de l'attributif (verbe) auquel il est lié par une préposition qui indique le rapport qu'il y a entre l'un et l'autre. » (Girault-Duvivier, 1811 [1840], p. 1151)

(82) « [le complément] exprimant un rapport de l'action au terme où elle aboutit : donner à quelqu'un; ou à celui d'où elle part : recevoir une lettre de son ami; et alors il est précédé, pour cette raison, d'une préposition : on l'appelle complément indirect. » (Landais, 1835, p. 393a) « Ce régime est précédé de la préposition $\grave{a}$, s'il s'agit du but où tend l'action; et de la préposition de, s'il s'agit du terme d'où cette action vient ou commence.»(Landais, 1835, p. 518a)

(83) «Ce que nous appelons communément régime indirect, et que d'autres appèlent plus logiquement le terminatif, [...] comme dans : il va à Paris, il entre dans sa chambre, il se tourne vers vous, ne correspond pas directement au datif des Latins, quoique ce soit pour nous comme pour eux le même cas logique, le but et la fin de l'action. » (Vanier 1836, p. 130)

Vanier (1836) souligne que quelques grammairiens modernes « opposent le complément éloigné, le point d'où part l'action; et ils appèlent circonstantiel tout ce qui n'est ni sujet de l'action, ni objet de l'action, ni terme de l'action. » (p. 131).

D'autres séquences $<$ Préposition $X>$ sont considérées comme des compléments circonstanciels. Si leur équivalence paradigmatique avec un adverbe est mise en avant, c'est la notion sémantique de circonstance qui l'emporte.

(84) «Ce que l'on emploie à exposer, soit la manière d'être de l'Attributif (verbe), soit la circonstance dans laquelle il a lieu, forme un cinquième membre ${ }^{2}$ que l'on nomme Circonstanciel; les mots qui expriment cette manière d'être ou cette circonstance sont ou des adverbes, ou des expressions adverbiales, ou quelque autre expression marquant une circonstance de temps, de lieu, d'action. » (Girault-Duvivier, 1811 [1840], T2, pp. 1151-1152)

(85) «Vous paissez dans nos champs, sans souci, sans alarmes. [...] Dans nos champs, voilà une circonstance de l'action. [...] Ces sortes de sens sont souvent exprimés en un seul mot, qu'on appelle adverbe. Sans souci [...] complément cirocnstanciel. » (Girault-Duvivier, 1811 [1840], T2, p. 1160)

L'équivalence avec un adverbe a amené la dénomination de complément adverbial, qui n'a pas remporté un franc succès. On la trouve chez Boniface (1829 [1843]), qui, sur cette base, scinde les compléments liés au verbe en compléments directs, indirects et adverbiaux. Un syntagme prépositionnel sera analysé comme complément adverbial s'il répond à une liste de questions spécifiques, sorties de la liste que la majorité des grammariens utilisent pour trouver un complément indirect.

(86) «Dans Il parle SAGEMENT, il viendra CE SOIR, ICI, POUR AFFAIRE, les compléments des verbes parle, viendra, les modifient par des idées de manière, de temps, de lieu de motif; ces compléments et leurs analogues sont appelés COMPLÉMENTS ADVERBIAUX, parce que, généralement, ce sont des adverbes, ou des expressions équivalentes. » (Boniface 1829 [1843], p. 30) «Le complément adverbial répond à toute question autre que celle du complément direct et du complément indirect. Les principales questions sont : quand? comment? où ? d'où ? par où ? pour quel motif ? (Boniface 1829 [1843], pp. 30-31) «L'homme travaille avec peine; Alexandre mourut au sein des plaisirs. » [compl adverbial] (Boniface 1829 [1843], p. 114) 
Les autres ouvrages qui usent du circonstanciel (Noël et Chapsal 1827, Thiel 1831) en font un complément prenant place au sein de l'analyse logique uniquement.

(87) «Le complément circonstanciel se joint au sujet ou à l'attribut pour en exprimer quelque circonstance; il est exprimé ou par un adverbe ou par un régime indirect; faisant l'office d'un adverbe. Parler beaucoup est le moyen de dire des sottises. Le temps passe promptement. Agir sans réflexion est le fait d'un insensé. Le mérite modeste plaît en tout temps. [...] Sans réflexion, est le complément circonstanciel du sujet agir, et en tout temps, celui de l'attribut plaisant. » (Noël et Chapsal, LAL, 1827 [1832], p. 5, par. 18)

(88) «Une phrase peut renfermer des mots qui ne fassent partie ni du sujet ni de l'attribut, par exemple : sans une attention soutenue, vous parviendrez difficilement à acquérir des connaissances solides. [...] L[a] préposition sans [...] ne li[e] à aucun mot ni du sujet ni de l'attribut, le complément qui le suit, et qui achève le sens général en y ajoutant quelques circonstances. Ces compléments sont appelés généraux ou circonstanciels. » (Thiel, 1831 [1836], p. 65)

La notion de complément circonstanciel semble encore peu consolidée et surtout utilisée avec modération en analyse logique. Seuls les adverbes, clairement identifiables, reçoivent systématiquement la fonction logique de complément circonstanciel chez les tenants de la première grammaire scolaire. Les compléments introduits par une préposition et pouvant être remplacés par un adverbe sont, quant à eux, très souvent classés comme des compléments indirects sur le plan logique alors qu'ils devraient, en principe, y être classés comme compléments circonstanciels. Ainsi, des compléments indirects grammaticaux marquant le lieu, et pouvant donc être remplacés par l'adverbe là, ne sont souvent pas considérés comme des compléments circonstanciels sur le plan logique. Dans Les grandes et belles pensées viennent du cœur (Noël et Chapsal, 1827[1832], p. 37), du cœur est un complément indirect grammatical du verbe viennent; il devrait être, dans l'analyse logique, une fois la phrase reformulée en Les grandes et belles pensées sont venant du cœur, un complément circonstanciel de l'attribut venant. Or, il est analysé comme un complément indirect de celui-ci. Il est également étonnant de constater que sans réflexion reçoit la fonction de complément circonstanciel dans Agir sans réflexion est le fait d'un insensé (Noël et Chapsal, 1827[1832], p. 5), tandis que avec réflexion reçoit celle de complément indirect dans Agir avec réflexion est le fait du sage (Noël et Chapsal, 1827[1832], LAL, p. 34). Il y a là un manque de cohérence que le vocabulaire ne peut expliquer seul : sans réflexion peut être remplacé par l'adverbe inconsidérément et obtient donc la fonction de complément circonstanciel; n'y aurait-il pas d'équivalents adverbiaux pour l'expression avec réflexion? On pense pourtant à raisonnablement, sagement, prudemment. Et cela est d'autant plus frappant que la définition du complément circonstanciel insiste bien sur le fait qu'il s'agit simplement pour le complément indirect grammatical de «faire office d'adverbe » (Noël et Chapsal, 1827[1832], p. 5), et non pas de pouvoir être remplacé exactement par un adverbe.

L'analyse des constructions verbe - syntagme prépositionnel présente un angle d'approche qui se distingue de celui adopté pour les autres constructions. Cette fois, l'analyse prend avant tout place dans le domaine de la signification, sans pour autant couper les liens avec l'analyse grammaticale. Si les questions sémantiques en qui? et en quoi? sont utilisées et permettent d'envisager la complétude du sens, l'insistance sur les prépositions qui les accompagnent montre non seulement l'importance encore accordée à l'analyse grammaticale linéaire, mais aussi l'absence de restriction sur la signification du complément.

\section{8 À propos de quelques compléments indirects des verbes}

Les compléments prépositionnels qu'il est intéressant de relever dans cet article sont de trois types : les compléments de lieu, les compléments des verbes passifs et les compléments effaçables. Tous ces compléments seront voués à une réanalyse au cours du XIX ${ }^{\mathrm{e}}$ et du $\mathrm{XX}^{\mathrm{e}}$ siècle. Ils constituent dès lors des marqueurs de périodisation dans l'histoire de la grammaire française. 
Les compléments prépositionnels indiquant un lieu sont, bien sûr, analysés comme des régimes indirects ou des compléments indirects. De nombreux grammairiens consignent des exemples de ce type. On y décèle avant tout des compléments en de (venir de, sortir de, s'éloigner de) ${ }^{3}$, en à (aller à, passer à, aboutir à $)^{4}$, en par (voyager par, passer par, venir par) ${ }^{5}$, en sur (tomber sur, régner sur, marcher sur) ${ }^{6}$, en dans (tomber dans, répandre dans, nager dans) ${ }^{7}$, et également, mais moins fréquemment, des compléments en vers (aller vers $)^{8}$ ou en en (aller en, monter en $)^{9}$.

Les compléments prépositionnels des verbes passifs sont analysés comme des régimes ou des compléments du verbe, et parfois nommés précisément régimes indirects ou compléments indirects.

(89) «Le verbe passif a un régime indirect formé par la préposition de ou par.» (Clouzet, 1824, $3^{\mathrm{e}}$ tableau, colonne 3 )

(90) «Les verbes passifs prennent devant leur régime indirect de [...] et par [...].» (M.C.A., 1825, p. 42)

(91) «Le sujet du verbe actif devient, pour ce même verbe au passif, un complément indirect particulier, qui lui est uni au moyen des prépositions par ou de [...]. » (Thiel 1831 [1836], p. 64)

(92) «Les verbes passifs ont leurs régimes indirects formés par la préposition de et par.» (Clouzet 1835, p. 134)

(93) «Tout verbe passif est formé d'un verbe actif, en prenant le sujet pour régime indirect et le régime direct pour sujet. » (D'Ablancourt, 1838, p. 22)

Les constructions suivantes apparaissent dans les grammaires du corpus au titre de régimes (indirects) ou de compléments (indirects) du verbe passif : des compléments en de (être aimé de, être méprisé de, être estimé de, être connu de, être adoré de) et des compléments en par (être frappé par, être trompé par, être fait par, être conquis par, être approuvé par, être sauvé par, être puni par, être mangé par, être fondé par, être donné par, être reçu par, être vaincu par, être embelli par, être gouverné par, être battu par, être fortifié par) ${ }^{10}$.

Les compléments prépositionnels périphériques, c'est-à-dire supprimables sans aucune modification profonde de sens, sont également classés comme des compléments ou régimes indirects : travailler pour la gloire (Noël et Chapsal, LAG, 1827 [1871], p. 5, par. 13); Richelieu fut craint des grands pendant toute sa vie; Le sage jouit avec modération des plaisirs du monde. (Noël et Chapsal, LAG, 1827 [1871], p. 67, ex XIII); Venir pour jouer; Courir afin d'atteindre (Thiel 1831, p. 64).

Les trois types de compléments prépositionnels que nous venons de passer en revue montrent que la période de la première grammaire scolaire se caractérise par une uniformité de traitement de ces structures. L'analyse de la complétude du sens s'efface derrière l'approche linéaire de la phrase. La présence d'une préposition donne invariablement lieu à un complément ou régime indirect.

\section{Conclusion}

Au cours de la première moitié du $\mathrm{XIX}^{\mathrm{e}}$ siècle, la préposition est vue comme un élément intervenant au sein d'une relation ternaire $<\mathrm{X}$ Préposition $\mathrm{X}>$. Certains grammairiens dépassent déjà l'analyse linéaire et agglomèrent la préposition avec l'élément qu'elle introduit. De manière générale, les analyses prennent place tant sur le plan grammatical que sur celui de la signification (c'est-à-dire de la complétude du sens).

Les séquences faisant intervenir un adjectif, un substantif ou un adverbe suivi d'un syntagme prépositionnel sont abordées par les grammairiens du corpus sous l'angle syntaxique. Au contraire, l'analyse des syntagmes prépositionnels apparaissant après un verbe montre une approche plus sémantique qui repose sur l'utilisation des questions. Cependant, la part de la préposition au sein de ces séquences est non négligeable, ce qui est l'indice d'un poids important accordé à la structure linéaire de l'analyse. Aucune restriction de sens n'est imposée à l'époque sur les compléments indirects, excepté chez quelques auteurs qui distinguent les compléments circonstanciels (de lieu et de temps notamment, paraphrasables par des adverbes) des compléments indirects. La distinction prend place sur le plan grammatical (Girault-Duvivier 1811) ou sur le plan logique (Noël et Chapsal 1827; Thiers 1831). 
Si les grammaires de la première moitié du XIX ${ }^{\mathrm{e}}$ siècle envisagent les syntagmes prépositionnels sous des angles un peu différents, elles se caractérisent néanmoins par une forte unité de traitement des constructions prépositionnelles. Elles proposent ainsi une analyse où la sémantique des compléments (complément de possession, de lieu, de temps, terme de l'action, origine de celle-ci, etc.) n'intervient encore que périphériquement. Il est alors possible, pour la majorité des grammaires, de traiter uniformément comme des compléments (indirects) la maison de mon père, content de ses enfants, nuire à quelqu'un, aller à Lyon, combattre pour la gloire, être battu par quelqu'un. En effet, la préposition occupe encore à cette époque une position clé au sein d'une analyse encore très linéaire.

(94) « c'est par elle [la préposition] que l'on connaît le régime indirect. » (Guillon, 1818, p. 118)

\section{Références bibliographiques}

Bescherelle aîné, L.-N., Bescherelle jeune, H.-H. et Litais de Gaux (1834 [1835-1836]). Grammaire Nationale ou Grammaire de Voltaire, de Racine, de Fénelon, de J.-J. Rousseau, de Buffon, de Bernardin de Saint-Pierre, de Chateaubriand, de Lamartine, et de tous les écrivains les plus distingués de la France. Renfermant plus de cent mille exemples qui servent à fonder les règles et constituent le code la langue française; ouvrage éminemment classique publié sous les auspices de MM. Casimir de la Vigne, de Jouy, Villemain, Tissot, Nodier, De Gérando, R. Jouhanneau, Boniface, Lévi et Sabatier. Paris : Bourgeois-Maze.

Bescherelle aîné, L.-N., Bescherelle jeune, H.-H. et Litais de Gaux (1834 [1852]). Grammaire Nationale ou Grammaire de Voltaire, de Racine, de Bossuet, de Fénelon, de J.-J. Rousseau, de Buffon, de Bernardin de SaintPierre, de Chateaubriand, de casimir delavigne, et de tous les écrivains les plus distingués de la France. Renfermant plus de cent mille exemples qui servent à fonder les règles et forment une espèce de panorama où se déroule notre langue telle que la Nation l'a faite, telle qu'elle doit la parler; ouvrage éminemment classique destiné à dévoiler le mécanisme et le génie de la langue française. Paris : Simon, Garnier Frères.

Bessière, A. (1834). Grammaire française. Paris : Delalain.

Boinvilliers, J. E. J. F. (1802). Grammaire raisonée ou Cours théorique et pratique de la langue française, a l'usage des personnes qui veulent connaitre et mettre en pratique non seulement les regles avouées depuis longtemps par les plus habiles grammairiens, mais encore des règles tout-à-fait neuves ou peu connues. Paris : Barbou.

Boinvilliers, J. E. J. F. (1802 [1818]). Grammaire raisonnée ou Cours théorique et analytique de la langue française, où sont renfermés non seulement les principes avoués depuis long-temps par les plus sçavants Grammairiens, mais encore des règles, les unes peu communes, les autres tout-à-fait neuves. Tomes 1 et 2. Paris: Auguste Delalain.

Boniface, A. (1829 [1843]). Grammaire française méthodique et raisonnée, rédigée d'après un nouveau plan, et fondée sur un grand nombre de faits et sur l'autorité des grammairiens les plus connus. Paris : Delalain.

Chervel, A. (1977). Et il fallut apprendre à écrire à tous les petits Français. Histoire de la grammaire scolaire, Paris : Payot.

Chervel, A. (1979). Rhétorique et grammaire, petite histoire du circonstanciel. Langue française, $n^{\circ} 41$, numéro spécial sur la grammaire traditionnelle, 5-19.

Clouzet, P. - A. (1824). De l'analyse grammaticale. Bordeaux : Suwerinck.

Clouzet, P. - A. (1835). Grammaire française sur un plan entièrement nouveau, divisé en cinq parties : conjugaison, analyse grammaticale, analyse logique, orthographe, et orthologie. Bordeaux: chez l'Auteur et ches les principaux libraires.

Cocquempot, J.-B. (1834). Éléments de la grammaire française de C.-F. Lhomond, avec des exercices gradués sur chaque règle. Paris : Delalain.

Coffigny, C. (1828). Élémens de la grammaire française, par demandes et par réponses, d'après les auteurs le splus accrédités. Commerci : Denis.

Cornillac, Ch. (1833). Grammaire française en tableaux, nouvelle méthode qui en facilite l'étude. Chatillon-surSeine : Charles Cornillac. 
Delvart, C. A. (1828). Cours théorique et pratique de grammaire, à l'usage des pensionnats et des personnes qui veulent apprendre la langue sans maître; contenant toutes le srègles que renferment les Grammaires les plus estimées et les plsu étendues, avec de nombreux exercices sur chacune de ces règles, et en outre un dictionnaire complet du régime des verbes. Paris : Le Normant, N. Pichard, Bocquet, Mongie, et les principaux libraires.

Farez, E. (1832). Nouvel abrégé de la grammaire française. Cambrai : Lesne-Daloin.

Fournier, M. (1801 [1827]). La Langue française et l'orthographe enseignées par principes et en vingt-quatre leçons, ou Grammaire française, à l'aide de laquelle on peut seul, et sans le secours d'aucun maître, apprendre à parler et à écrire correctement cette langue. Paris : Germain Mathiot.

Gaillard, F. (1810 [1820]). Premiers éléments de la grammaire françoise. Ouvrage adopté pour la classe d'orthographe du Collége de Genève. Genève : chez l'Auteur.

Gaultier, L. (1829). Élémens de grammaire française, extraits de la grammaire de l'Abbé Gaultier, nouvelle édition entièrement refondue et considérablement augmentée par De Blignière, demoyencourt, Ducros (de Sixt) et Le Clerc aîné, ses élèves. Première édition. Paris : Jules Renouard.

Gilard, M. (1837). Grammaire pratique, analytique et théorique de la langue française, divisée en trois parties. Première partei : prononciation grammaticale et prosodique. Deuxième partie : classification et nature des mots démontrée par l'analyse grammaticale subdivisée d'après le plan méthodique de l'ouvrage; théorie nouvelle des verbes. Troisième partie : participes; accord, significations, orthographe et emploi des mots; nouveau moyen d'apprendre par l'analyse logique la construction des mots, des propositions et des phrases; inversions; ellipses; pléonasmes; gallicismes; précis de versification. Paris : Daubrée.

Girault-Duvivier, Ch. P. (1811 [1840]). Grammaire des Grammaires ou Analyse Raisonnée des meilleurs traités sur la langue française, Ouvrage mis par l'Université au nombre des Livres à donner en prix dans les Colléges, Et reconnu par l'Académie française comme indispensable à ses travaux et utile à la littérature en général. Paris : Cotelle.

Guillon, Ch. (1818). Méthode facile pour apprendre promptement l'orthographe et les premiers principes de grammaire française. Grenoble : Allier.

Landais, N. (1835 [1856]). Grammaire Générale des Grammaires Françaises présentant la solution analytique, raisonnée et logique de toutes les questions grammaticales anciennes et modernes. Paris : Didier.

Lhomond, C. F. (1811). Grammaire françoise de Lhomond, A l'usage des lycées et des institutions; Revue, corrigée, et augmentée par Charles-Constant Letellier. Paris : Le Prieur, belin et chez l'Auteur.

Le Tellier, Ch.-C. (1816 [1822]). Grammaire des commençants, contenant la connoissance des diverses espèces de mots employés dans la langue françoise, la concordance de ces mots dans la phrase, un traité d'orthographe, et une méthode d'analyse grammaticale; par demandes et par réponses. Paris : Constant Le Tellier Fils.

Lemare, P. A. (1807 [1819]). Cours de langue française en six parties: Idéologie, Lexicographie, Prononciation, Syntaxe, Construction, Ponctuation; où 5000 exemples, pris dans Pascal, Bossuet, Fénélon, Racine, La Fontaine et autres classiques, servent à fonder les règles et présentent des modèles de tous les genres et de tous les styles. Tomes 1 et 2. Paris : Henry Grand, Bachelier, Bechet, brunot, Madame Huzard.

M. C. A. (1825). Petite grammaire des grammaires ou système synoptique de grammaire française. Paris : MaireNyon.

Noël, F. J. M. et Chapsal, Ch. P. (1823 [1831]). Nouvelle grammaire française sur un plan très-méthodique avec de nombreux exercices d'orthographe, de syntaxe et de ponctuation tirés des meilleurs auteurs, et distribués dans l'ordre des règles. Bruxelles : Hauman et comp.

Noël, F. J. M. et Chapsal, Ch. P. (1827 [1832]). Leçons d'analyse logique contenant des préceptes sur l'art d'analyser et des exercices et des sujets d'analyse logique gradués et calqués sur les préceptes; suivies d'un programme de questions sur la seconde partie de la Nouvelle grammaire française. Paris : Maire-Nyon, Roret, Delalain.

Noël, F. J. M. et Chapsal, Ch. P. (1827 [1871]). Leçons d'analyse grammaticale contenant des préceptes sur l'art d'analyser et des exercices et des sujets d'analyse grammaticale gradués et calqués sur les préceptes. Paris: Maire-Nyon, Roret, Hachette, Delalain.

Perrot D'Ablancourt, M. (1838). Nouvel abrégé de grammaire française, suivi d'un traité d'orthographe pratique, à l'aide duquel on peut apprendre à écrire convenablement et seul la plupart des mots de notre langue. Poitiers : Fradet. 
Restaut, P. (1732). Principes généraux et raisonnés de la grammaire françoise avec des observations sur l'orthographe, les accents, la ponctuation, et la prononciation: et un abrégé des règles de la versification française, dédiés à Monseigneur le Duc de Chartres. Nouvelle édition corrigée et augmentée. Paris : Le Gras, Lottin, Desaint, Chaubert.

Reymond, S. (1811). Leçons de grammaire française, par demandes et réponses, avec des remaques et des notes, suivies d'un Abrégé de Syntaxe, d'Analyse, de fautes contre la Langue, et des principaux mots homonymes. Lyon: Yvernault et Cabin; Paris : Brunot-Labbé.

Thiel, A. (1831 [1836]). Leçons élémentaires méthodiques et pratiques de grammaire française. Metz: Madame Thiel; Paris : Hachette.

Vanier, V.-A. (1836). Dictionnaire grammatical, critique et philosophique de la langue française. Paris : chez l'Auteur, Brunot-Labbé, Delalain, Dupont, Janet et Cotelle.

\footnotetext{
${ }^{1}$ Nous adoptons ici l'analyse de l'époque.

${ }^{2}$ Les autres membres de la phrase sont le sujet, l'attributif (verbe), l'objet (représenté par le régime direct) et le terme (représenté par le régime indirect).
}

${ }^{3}$ Venir de la maison (Boinvilliers 1802, T1, p. 230); Rien ne suffit aux gens qui nous viennent de Rome (Lemare 1804 [1835], T1, par. 1003); A peine nous sortions des portes de Trézène (Girault-Duvivier 1811 [1840], p. 1155); Il revient de l'armée (Gaillard 1810 [1820], p. 93); Je m'éloigne de Paris, Je viens de l'Amérique, Je sors du Jardin (Gaultier 1829, p. 23).

${ }^{4}$ Aller à la campagne (Guillon 1818, p. 98); J'ai passé à Toul (Coffigny 1828, p. 82); Un champ qui aboutit au grand chemin (Delvart 1828, p. 263); Aller au but (Thiel 1831, p. 64).

${ }^{5}$ Voyager par la campagne (Boinvilliers 1802, T1, p. 230); J'ai passé par la prairie [...] (Coffigny 1828, p. 82); Passer par la ville (Landais 1835, p. 393a); Il vient par ici (Vanier 1836, p. 682.

${ }^{6}$ Tomber sur quelque chose (Reymond 1811, p. 107); Régner sur une nation brave (Girault-Duvivier 1811 [1840], T1, p. 678); Marcher sur la terre (Thiel 1831, p. 64); Il marche sur la bordure (Vanier 1836, p. 682).

${ }^{7}$ Tomber dans la misère (Girault-Duvivier 1811 [1840], T1, p. 678); Tomber dans l'eau (Thiel 1831, p. 64); Ils répandront dans Ithaque et dans les pays voisins le prochain retour d'Ulysse (Bescherelle 1834 [1852], p. 426); Il éternue dans son mouchoir; il nage dans le bassin (Vanier 1836, p. 682).

${ }^{8}$ Aller vers le but (Thiel 1831, p. 64).

${ }^{9}$ Anacharsis alla en Grèce (Noël et Chapsal, LAG, 1823 [1871], p. 58, exercice X); Monter en voiture (Clouzet 1835 , p. 133).

${ }^{10}$ Les plus hautes montagnes sont frappées par la foudre (Boinvilliers 1802, T1, p. 16); Exemples : un enfant doux et docile est aimé de ses parents. J'ai été trompé par l'homme que je regardais comme mon meilleur ami. (Le Tellier 1807 [1811], p. 144); Un jeune homme ignorant et orgueilleux est méprisé DE tous ceux qui le connoissent. - La première opération de la fistule a été faite sur Louis XIV, PAR le célèbre Mareschal. (Girault-Duvivier 1811 [1840], T1, p. 677); L'honnête homme est estimé de tout le monde. Une grande partie de la terre a été conquise par les Romains. Votre conduite a été approuvée d'une commune voix par toutes les personnes sages et éclairées. (Noël et Chapsal, NGF, 1823 [1831], p. 140); Il a été sauvé par mes soins (Fournier 1827, $3^{\mathrm{e}}$ éd., p. 67) Il sera puni par le maître. (Fournier 1827, $3^{\mathrm{e}}$ éd., p. 90); Un enfant sage est aimé de ses parents. La souris est mangée par le chat. (Coffigny 1828, p. 81); L'honnête homme est estimé de tout le monde. Une grande partie de la terre a été conquise par les Romains. Rome a été fondée par Romulus. Il n'est aimé de personne. (Delvart 1828, p. 390); La récompense est donnée par le roi. Les éloges sont reçus par l'enfant studieux. Le père est aimé de son fils. (Thiel 1831 [1836], p. 64); L'enfant studieux est aimé de ses maîtres; Darius fut vaincu par Alexandre. (Cornillac 1833, section Remarques sur les verbes); La jeunesse est embellie par les grâces. (Bescherelle 1834 [1852], p. 492) Il veut être connu et adoré de sa créature. Les rats sont gouvernés par la raison d'état. (Bescherelle 1834 [1852], p. 503); La souris est mangée par le chat. Un enfant sage est aimé de ses parents. (Cocquempot 1834, p. 76); Il est aimé de ses parents. Il a été battu par son maître. (Clouzet 1835, p. 134); Ce pilotis est fortifié par des branches (Gilard 1837, p. 82); L'homme de bien est aimé de Dieu. (D'Ablancourt, 1838, p. 22). 